\title{
Nota \\ PARASITÓIDES DE Cerconota anonella (Sepp., 1830) (Lep.: Oecophoridae) EM GRAVIOLEIRA (Annona muricata L.)
}

\author{
Sônia Maria Forti Broglio-Micheletti; Evôneo Berti-Filho²* \\ ${ }^{1}$ Depto. de Fitotecnia e Fitossanidade - CECA/UFAL, BR 104, Km 85 - CEP: 57100-000 - Rio Largo, AL. \\ ${ }^{2}$ Depto. de Entomologia, Fitopatologia e Zoologia Agrícola - USP/ESALQ, C.P. 9 - CEP: 13418-900 - Piracicaba, SP. \\ *Autor correspondente<eberti@carpa.ciagri.usp.br>
}

\begin{abstract}
RESUMO: A quantificação de inimigos naturais presentes em uma cultura é importante, porque influi nas decisões a serem tomadas em relação ao Manejo Integrado de Pragas. Assim, este trabalho teve por objetivos identificar e quantificar gêneros de parasitóides de Cerconota anonella (Sepp.) coletados na cultura de graviola entre março de 1997 e abril de 1998 em Maceió, AL (lat.95, long. 35W). O parasitismo foi determinado pela relação entre o número de formas biológicas dos inimigos naturais e o de formas biológicas da praga e dos parasitóides. Os insetos, por ordem de incidência, foram: Apanteles sp. (Hymenoptera: Braconidae), Rhysipolis sp. (Hymenoptera: Braconidae) e Xiphosomella sp. (Hymenoptera: Ichneumonidae). Apanteles sp. predominou durante o período. Os menores percentuais de parasitismo total foram observados no mês de outubro de 1997 $(6,60 \%)$ e os maiores em janeiro de 1998 ( $80,27 \%)$, resultando em um parasitismo médio anual de $38,1 \%$. Palavras-chave: Insecta, Hymenoptera, Braconidae, Ichneumonidae, inimigo natural
\end{abstract}

\section{PARASITOIDS OF Cerconota anonella (Sepp., 1830) (Lep.: Oecophoridae) IN SOURSOP (Annona muricata L.)}

\begin{abstract}
The quantification of natural enemies actually living within a given crop is very important, because it helps in pest control decisions. Therefore a survey of natural enemies of Cerconota anonella (Sepp.) was carried out in a soursop crop between March,1997 and April,1998 at Maceió, AL (lat.9S, long. 35W), Brazil. The parasitism was defined by the ratio: the number biological forms of natural enemies and the biological forms of pests and parasitoids. The insects observed, in order of incidence, were: Apanteles sp. (Hymenoptera: Braconidae), Rhysipolis sp. (Hymenoptera: Braconidae) e Xiphosomella sp. (Hymenoptera: Ichneumonidae) . The lowest percentage of parasitism was found in October 1997 (6.6\%) and the highest one in January 1998 (80.27\%), with an annual average of $38.1 \%$.
\end{abstract}

Key words: Insecta, Hymenoptera, Braconidae, Ichneumonidae, natural enemy

\section{INTRODUÇÃO}

$O$ recente desenvolvimento de mercado para as frutas tropicais no Brasil e no mundo reativou o comércio de polpas congeladas para sucos, sorvetes, geléias, etc. A polpa da graviola está entre as mais solicitadas nos mercados do Nordeste e este interesse tem crescido bastante, sobretudo no Centro-Sul do Brasil (Lemos, 1996). Apesar das condições ecológicas favoráveis ao seu cultivo, os problemas fitossanitários, especialmente as pragas, vêm desestimulando o estabelecimento de plantios comerciais no País. A broca-do-fruto, Cerconota anonella (Sepp.) (Lepidoptera: Oecophoridae) é considerada uma das mais sérias pragas da gravioleira pelos danos expressivos que causa à cultura, tanto no Brasil como em outras regiões do mundo. A maioria das referências bibliográficas limita-se a citar a ocorrência de parasitóides desta praga, sem contudo quantificar a importância dos mesmos. Nuñez L. \& De la Cruz (1982) observaram na Colômbia os seguintes inimigos naturais de C. anonella: Chrysopa sp. (Neuroptera: Chrysopidae), Baccha sp. (Diptera: Syrphidae), Cycloneda sanguinea Linné, Hippodamia convergens Guerin e Curinus sp. (Coleoptera: Coccinellidae); Zelus sp. (Hemiptera: Reduviidae) e Polistes sp. (Hymenoptera: Vespidae). Na Venezuela, Boscán de Martínez \& Godoy (1983) relataram a ocorrência de Apanteles stenomae Muesebeck (Hymenoptera: Braconidae) atacando larvas de $C$. anonella, tendo o parasitismo variado de $2,81 \%$ a $51,61 \%$, conforme a localidade estudada. Observaram também o parasito larval do gênero Xyphosomella (Hymenoptera: Ichneumonidae), porém sem quantificar o parasitismo. Bustillo \& Peña (1992) observaram, na Colômbia e no Equador, o parasitóide larval Apanteles sp. e um gênero desconhecido da subfamília Rogadinae, alcançando para o primeiro, um parasitismo de 2 a $5 \%$ na Colômbia e $2 \%$ no Equador.

Elaborou-se este trabalho com o objetivo de quantificar gêneros de parasitóides de $C$. anonella presentes em pomar de graviola. 


\section{MATERIAL E MÉTODOS}

Os frutos de graviola atacados por $C$. anonella foram coletados quinzenalmente, de março de 1997 a abril de 1998, no Sítio Aldeia Verde, localizado em Maceió - AL (lat.9 ${ }^{\circ}$, 40 S, long. $35^{\circ}, 42 \mathrm{~W}$ ). No laboratório de Entomologia do Centro de Ciências Agrárias da Universidade Federal de Alagoas, parte dos frutos colhidos quinzenalmente foi individualizada e colocada em recipientes de plástico telados. Manteve-se este material em condições ambientais (Temperatura Média 26,0 $\pm 1,33$, Umidade Relativa $78,18 \% \pm 2,06$ ). Diariamente observava-se a emergência dos parasitóides e da praga. Os frutos remanescentes da coleta (variaram de 15 a 22) foram dissecados para observação das formas imaturas tanto da praga (larvas parasitadas ou não e pupas) como dos inimigos naturais (pupas). Às larvas eram fornecidos pedaços de graviola não madura como alimento, substituídos em dias alternados.

Os parasitóides foram alimentados com solução de mel a $50 \%$ e, quando mortos, colocados em frascos contendo álcool a $70 \%$ para a identificação taxonômica.

O parasitismo foi determinado pela relação entre o número de formas biológicas do inimigo natural (pupas + adultos + larvas parasitadas) e o número total de formas biológicas da praga (larvas + pupas +adultos) + do inimigo natural. (pupas + adultos + larvas parasitadas).

Com os resultados obtidos, calcularam-se os índices faunísticos de freqüência e constância (Silveira Neto et al., 1976), segundo os quais uma espécie pode ser constante (presente em mais de $50 \%$ das coletas), acessória (presente em $25-50 \%$ das coletas) ou acidental (presente em menos de $25 \%$ das coletas).

\section{RESULTADOS E DISCUSSÃO}

Os inimigos naturais foram Apanteles sp. (Hymenoptera: Braconidae, Microgastrinae), Rhysipolis sp. (Hymenoptera: Braconidae, Hormiinae) e Xiphosomella sp. (Hymenoptera: Ichneumonidae, Cremastinae), registrandose o parasitismo (\%) desses insetos (Figura 1). O total de formas biológicas da praga (larva, pupa e adulto) foi de 2019 e as dos inimigos naturais 991 (pupas, larvas parasitadas e adultos), resultando em um parasitismo total de $32,92 \%$. Dentre os inimigos naturais observados, $74,07 \%$ eram Apanteles sp., considerada constante por estar presente em $96,55 \%$ das coletas; $24,42 \%$ eram Rhysipolis sp. classificada como espécie acessória ocorrendo em $48,28 \%$ das coletas e $1,51 \%$ eram Xiphosomella sp. espécie acidental aparecendo em $24,14 \%$ das amostras. O parasitismo por Apanteles sp. predominou nos períodos de agosto-setembro/1997 e março-abril/1998, por Rhysipolis sp. janeiro-abril/1998 e Xiphosomella sp. agosto/setembro/1997. O menor parasitismo total (\%) verificou-se em outubro/1997, tendo predominado em janeiro-abril/1998.

As pupas de Apanteles sp. ocorreram individualizadas, sempre internamente e próximas à região da casca dos frutos. Estes insetos são endoparasitóides solitários de larvas. As pupas de Rhysipolis sp. foram verificadas em grupos, tendo 4 a 6 casulos cada um , são endoparasitóides gregários. No caso de Xiphosomella sp. somente foram observados adultos.

Pretende-se criar massalmente Apanteles sp., para liberá-lo posteriormente em campo de graviola atacada por Cerconota anonella.

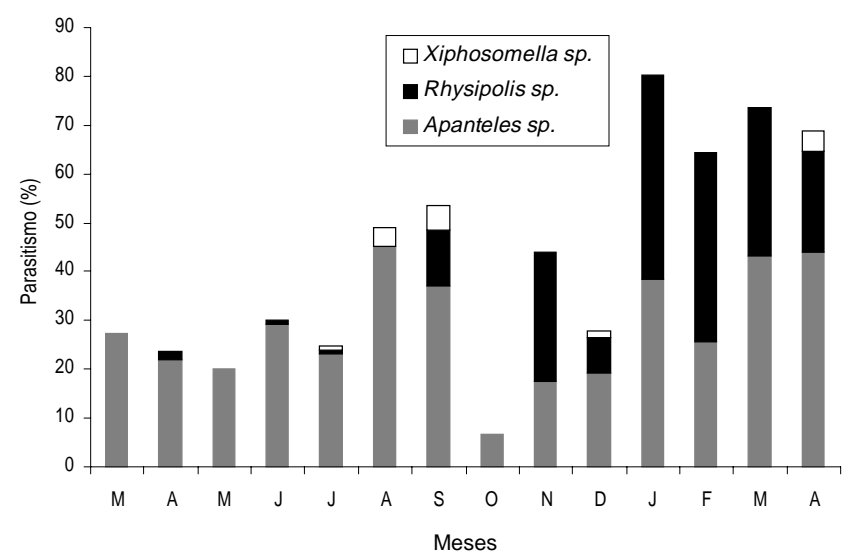

Figura 1 - Parasitismo mensal de Cerconota anonella por Apanteles sp., Rhysipolis sp. e Xiphosomella sp., em graviola. Sítio Aldeia Verde, Maceió, AL, março/1997 - abril/1998.

\section{CONCLUSÕES}

- Os gêneros de parasitóides de $C$. anonella em graviola são: Apanteles sp. e Rhysipolis sp. (Hymenoptera: Braconidae) e Xiphosomella sp. (Hymenoptera: Ichneumonidae).

- Apanteles sp. é o gênero do principal inimigo natural de larvas de $C$. anonella.

\section{AGRADECIMENTOS}

Aos funcionários: Roseane Maria Lins e Silva e $\mathrm{Eng}^{\circ}$ Agrônomo Antonio Jorge de Araújo Viveiros, CECA/ FIT/UFAL, pela ajuda na coleta do material. À CAPES/ PIDCT pelo auxílio financeiro.

\section{REFERÊNCIAS BIBLIOGRÁFICAS}

BOSCÁN de MARTÍNEZ, N.; GODOY. F.J. Enemigos naturales de Cerconota anonella Sepp. perforador del fruto de la guanabana (Annona muricata L.). Agronomia Tropical, v.33, p.155-161, 1983.

BUSTILLO, A.E.; PEÑA, J.E. Biology and control of the Annona fruit borer Cerconota anonella (Lepidoptera: Oecophoridae). Fruits, v.47, p.81-84, 1992.

LEMOS, E.E.P. de. Experimentos em micropropagação e organogênese na graviola (Annona muricata L.). Maceió: EDUFAL, 1996. 42p. (Série Apontamentos, 4).

NUÑEZ L., V.R.; DE LA CRUZ, J. Reconocimiento y descripcion de los principales insectos observados en cultivares de guanabano (Annona muricata L.) en el departamento del Valle. Acta Agronomica, v.32, p.45-51, 1982.

SILVEIRA NETO, S.; NAKANO, O.; BARBIN, D.; VILLA NOVA, N.A. Manual de ecologia dos insetos. São Paulo: Ceres, 1976. 419p.

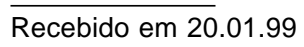

